\title{
PREVALÊNCIA DAS MALOCLUSÕES EM CRIANÇAS PRÉ-ESCOLARES NO MUNICÍPIO DE LAVRINHAS, SP
}

\author{
Eduardo César Werneck ${ }^{1}$, Fernanda Silva Mattos ${ }^{2}$, Márcio Garcia da Silva ${ }^{3}$, Renata Falchete do Prado ${ }^{4}$, \\ Gustavo Lopes Carvalho ${ }^{5}$, Adriano Marotta Araújo ${ }^{6}$
}

\footnotetext{
${ }^{1}$ Coordenador do Instituto de Ensino e Pesquisa de Cruzeiro (IEPC); ${ }^{2}$ Professora do curso de aperfeiçoamento - Instituto de Ensino e Pesquisa de Cruzeiro (IEPC); ${ }^{3}$ Professor do curso de especialização - Instituto de Ensino e Pesquisa de Cruzeiro (IEPC); ${ }^{4}$ Professora de Patologia do Departamento de Fisioterapia da Escola Superior de Cruzeiro Prefeito Hamilton Vieira Mendes; ${ }^{5}$ Aluno de especialização em ortodontia - Instituto de Ensino e Pesquisa de Cruzeiro (IEPC); ${ }^{6}$ Professor assistente doutor da Faculdade de Odontologia de São José dos Campos - UNESP
}

\section{RESUMO}

O objetivo deste trabalho foi verificar a prevalência das maloclusões em crianças pré-escolares, das escolas de Lavrinhas, SP, para se conhecer os problemas ortodônticos mais frequentes, permitindo a orientação adequada. Exames clínicos de 257 crianças foram realizados por meio da visualização dos arcos dentários em oclusão, relacionando-os aos critérios adotados para classificar as oclusões normais, os problemas transversais, verticais, sagitais e de espaço. Os dados obtidos foram tabulados e submetidos à análise estatística descritiva (\%) e teste de correlação de Pearson (5\%). Um alto índice de maloclusão $(91,05 \%)$ foi encontrado, além de correlação negativa $(r=-0,189)$ e estatisticamente significante $(p=0,002)$ entre cárie e diastema, e entre ausência de maloclusão e cáries $(r=-0,668 ; p=0,0001)$; e correlação positiva $(r=0,418)$ e estatisticamente significante entre Classe II e mordida aberta $(p=0,0001)$, atresia e mordida cruzada $(r=0,425 ; p=0,0001)$; atresia, mordida cruzada e mordida aberta $(r=0,235 ; p=0,0001)$ e Classe II, mordida aberta, atresia e cruzamento $(r=0,443 ; p=0,0001)$. Os resultados demonstraram a necessidade de instituição de tratamento ortodôntico, além de orientações adequadas de higienização, nutrição e da remoção de hábitos parafuncionais. A integralização da ortodontia nos serviços públicos de saúde é importante e, quando iniciada em fase precoce, possui custos mais baixos viabilizando um possível tratamento.

Palavras-chave: ortodontia preventiva, maloclusão, epidemiologia.

\section{MALOCCLUSION PREVALENCE IN PRESCHOOLERS' CHILDREN INMUNICIPALITY OF LAVRINHAS, SP}

\begin{abstract}
The aim of this study was to verify the prevalence of malocclusion in all preschool aged children in the municipality of Lavrinhas, SP, to know the most common orthodontic problems thus allowing proper orientation. Clinical examinations of 257 children were performed by viewing the dental arches, relating them to the criteria used to classify normal occlusion, transversal, vertical, sagittal and space problems. Data were tabulated and submitted to descriptive statistical analysis (\%) and Pearson correlation test (0.05). A high incidence of malocclusion (91.05\%) was found as well as negative correlation $(r=-0.189)$ and statistically significant $(p=0.002)$ between cavity and diastema, and between the absence of malocclusion and caries $(r=-$ 0.668; $p=0.0001)$; and positive correlation $(r=0.418)$ and statistically significant association between open bite and Class II $(p=0.0001)$, atresia and crossbite $(r=0.425 ; p=0.0001)$; atresia, cross and open bite $(r=0.235 ; p=0.0001)$ and Class II, open bite, atresia and crosses $(r=0.443 ; p=0.0001)$. The results demonstrate the need for establishment of orthodontic treatment, and appropriate guidelines for hygiene, nutrition and the removal of deleterious habits. The payment of orthodontics in public health services is important and, when initiated at an early stage (preventive/interceptive), has much lower costs enabling a possible treatment.
\end{abstract}

Keywords: preventive orthodontics, malocclusion, epidemiology. 


\section{INTRODUÇÃO}

As maloclusões são desvios de normalidade das arcadas dentárias, do esqueleto facial ou de ambos, com reflexos variados nas diversas funções do aparelho estomatognático, assim como na aparência e auto-estima dos indivíduos afetados (BRESOLIN, 2000).

O conhecimento da situação de saúde bucal e prevalência de maloclusão de uma determinada região através de estudos epidemiológicos fornecem importantes dados para que se possam planejar medidas necessárias para a redução da incidência dos fatores desfavoráveis observados, além da interceptação dos problemas esqueléticos em idade adequada (RIBAS et al., 2004).

Sabe-se que a prevalência de problemas oclusais moderados ou severos no Brasil aos cinco anos é de 14,5\% (PROJETO SB BRASIL, 2004), enfatizando a necessidade de planejamento para o controle destas anomalias, por meio de tratamentos especializados gratuitos em redes públicas de atendimento.

Dentro deste contexto, a avaliação das oclusões para fins de saúde pública tem como principal objetivo determinar as características oclusais de determinada população para verificar as necessidades de planejamento dos recursos requeridos para sua realização, tais como capacidade de trabalho, habilidade, agilidade e materiais empregados, além de estimular 0 fornecimento do tratamento ortodôntico pelos serviços de saúde pública (FOSTER \& MENEZES, 1976).

A partir dos dados coletados é possível planejar, executar e avaliar ações de saúde, além de permitir comparações de prevalências em diferentes períodos de tempo e áreas geográficas (OLIVEIRA et al., 1998). Inúmeros trabalhos epidemiológicos têm sido publicados demonstrando altos índices de maloclusões (SEWARD, 1965; DE VIS et al., 1984; SILVA
FILHO et al., 1990; TIPTON \& RINCHUSE, 1990; KABUE et al., 1995; OLIVEIRA et al., 1998; NORMANDO et al., 1999; TOMITA et al., 2000; FRAZÃO et al., 2002; RIBAS et al., 2004; SADAKYO et al., 2004; MARQUES et al., 2005; THOMAZ \& VALENÇA, 2005; SULIANO et al., 2007; ALMEIDA-PEDRIN et al., 2008; ARASHIRO et al., 2009).

A padronização da classificação das maloclusões é um fator importante para uma comunicação universal. Assim, a utilização da classificação de Angle (1989) tende a facilitar o entendimento e clareza do trabalho, sendo a primeira definição clara e simples de oclusão normal. Angle (1989) afirma que os primeiros molares superiores são a chave da oclusão e os molares superiores e inferiores devem se relacionar de modo que a cúspide mesiovestibular do molar superior oclua no sulco vestibular do molar inferior, com os dentes dispostos em uma curva suave de oclusão.

Dessa forma, o objetivo deste trabalho foi verificar a prevalência das maloclusões em crianças em idade pré-escolar, em todas as escolas da cidade de Lavrinhas, São Paulo, Brasil, para se conhecer os problemas ortodônticos mais frequentes, permitindo que se faça um planejamento e orientação adequada.

\section{MÉTODOS}

Este projeto foi submetido ao Comitê de Ética em Pesquisa da Faculdade de Odontologia de São José dos Campos - UNESP e aprovado sob protocolo $n^{0}$ 019/2011.

Para a realização deste estudo, foram avaliadas 257 crianças sem distinção de raça e de classe social, de ambos os sexos, com idade de 4 a 7 anos, pertencentes a todas as escolas municipais de Lavrinhas, São Paulo, Brasil, cuja população é de 7002 habitantes (IBGE, 2010). Os exames foram conduzidos por dois examinadores previamente treinados e com a mesma linha de 
formação acadêmica, para obtenção de avaliação padronizada. O exame clínico de cada escolar foi realizado por meio da inspeção visual utilizandose espátulas de madeira descartáveis, nas salas de aula da própria escola, com boas condições de visão e iluminação natural. Cada aluno foi examinado por meio da visualização dos arcos dentários em abertura e em oclusão, relacionando-os aos critérios adotados para classificar as oclusões normais, os problemas transversais (atresia ou mordida cruzada), verticais (aberta ou profunda), sagitais (Classe I, II e III) e de espaço (relação entre espaço presente e espaço requerido).

Os dados obtidos foram anotados em ficha cadastral específica, identificando o sexo e as maloclusões encontradas. Os resultados foram tabulados e submetidos à análise estatística descritiva, com obtenção dos valores de porcentagem e teste de correlação de Pearson, com nível de significância de 0,05\%.

De acordo com a presença de maloclusões, os pais foram orientados para a necessidade de intervenção e/ou acompanhamento ortodôntico, além da orientação ao setor público responsável sobre as reais necessidades encontradas nas crianças do município para uma elaboração de futuro e possível plano de ação.

\section{RESULTADOS}

A prevalência de maloclusão foi de $91,05 \%$. Os dados encontrados na análise do exame intra-bucal das 257 crianças podem ser observados na Tabela 1.

Tabela 1. Prevalência das maloclusões encontradas (em números e porcentagem).

\begin{tabular}{|c|c|c|c|c|c|c|c|}
\hline Sexo & \multicolumn{4}{|c|}{ ESPAÇO } & \multicolumn{3}{|c|}{ TRANSVERSAL } \\
\hline$F$ & S/ Probl & Cárie & Diastema & Perda Severa & Atresia & Cruzada & S/ Probl \\
\hline $\begin{array}{cc}14 & 10 \\
8 & 9\end{array}$ & 163 & 53 & 31 & 10 & 128 & 39 & 129 \\
\hline \multirow[t]{5}{*}{257} & $63,42 \%$ & $20,62 \%$ & $12,06 \%$ & $3,89 \%$ & $49,80 \%$ & $15,17 \%$ & $50,19 \%$ \\
\hline & \multicolumn{4}{|c|}{ VERTICAL } & \multicolumn{3}{|c|}{ SAGITAL } \\
\hline & Topo & S/ Probl & Aberta & Profunda & CL I & CL II & CL III \\
\hline & 41 & 75 & 90 & 51 & 146 & 95 & 16 \\
\hline & $15,95 \%$ & $29,18 \%$ & $35,01 \%$ & $19,84 \%$ & $56,80 \%$ & $36,96 \%$ & $6,22 \%$ \\
\hline
\end{tabular}

Pôde-se verificar que, no sentido sagital, houve maior incidência de maloclusão de Classe I (56,80\%); no sentido transversal, a maior incidência ocorreu nas atresias (49,80\%); no sentido vertical, a mordida aberta foi a maloclusão mais freqüente $(35,01 \%)$; no que diz respeito às questões de espaço, observa-se que houve maior freqüência de cárie dentária, que consequentemente levará a uma perda de espaço (20,62\%).
Foi constatado que uma mesma criança possuía mais de uma maloclusão, como pode ser observado na tabela 1. Destacaram-se algumas maloclusões conjuntas. Assim, o teste estatístico (correlação de Pearson) foi aplicado para testar a existência de correlação entre tais maloclusões associadas ou não, apresentadas na Tabela 2. 
Tabela 2. Teste de correlação de Pearson e nível de significância (p).

\begin{tabular}{lcc}
\hline \multicolumn{1}{c}{ Problemas Correlacionados } & Correlação de Pearson (r) & Valor de $\mathbf{p}$ \\
\hline Mordida Profunda x Classe II & $-0,017$ & 0,783 \\
Diastema x Perda Severa & $-0,073$ & 0,242 \\
Cárie x Diastema & $-0,189$ & 0,002 \\
Ausência de maloclusão x Classe I & 0,093 & 0,138 \\
Classe II x Mordida aberta & 0,418 & 0,0001 \\
Atresia x Mordida cruzada & 0,425 & 0,0001 \\
Perda Severa x Mordida Cruzada & $-0,029$ & 0,640 \\
Ausência de Maloclusão x Cárie & $-0,668$ & 0,0001 \\
Mordida Aberta x Atresia x Mordida Cruzada & 0,235 & 0,0001 \\
Classe II x Mordida aberta x Atresia x Mordida & 0,425 & 0,0001 \\
Cruzada & & \\
\hline
\end{tabular}

Após a análise dos resultados, pôde ser observado correlação negativa e estatisticamente significante $(p=0,002)$ entre cáries e diastemas, ou seja, os escolares que possuíam diastemas não possuíam cáries que pudessem causar perda de espaço.

Ainda em relação à presença de cáries verificou-se que a ausência de maloclusão está correlacionada negativamente $(r=-0,668)$ com a presença de cárie, sendo estatisticamente significante $(p=0,0001)$. Assim nas crianças com cáries, havia maior prevalência de maloclusão.

Também foi observada correlação positiva e estatisticamente significante entre Classe II e mordida aberta $(p=0,0001)$, verificando-se a presença de Classe II quando ocorre a mordida aberta. Outro fator estatisticamente significante $(p=0,0001)$ foi a relação entre atresia e mordida cruzada, possuindo correlação positiva $(r=0,425)$, sendo que a observação da mordida cruzada implica na presença de atresia. Além desta, a correlação entre atresia, mordida cruzada e mordida aberta foi estatisticamente significante $(p=0,0001)$, e também a correlação entre Classe II, mordida aberta, atresia e mordida cruzada $(p=0,0001)$.
A prevalência de maloclusão neste estudo foi alta $(91,05 \%)$, mas coerente com outros trabalhos da literatura como Almeida et al. (2008) que encontraram a prevalência de 95,73\%. Outros estudos verificaram prevalência de 88,53\% (SILVA FILHO et al., 1990), 71,6\% (SADAKYO et al., 2004), 71,4\% (THOMAZ \& VALENÇA, 2005) e 87,4\% (ARASHIRO et al., 2009). Acredita-se que o fator principal para estas divergências encontra-se no fato da população brasileira possuir uma grande miscigenação que provoca maior taxa de maloclusão (RIBAS et al., 2004) e diferenças entre as diversas regiões.

Além disso, o nível sócio-econômico pode influenciar significativamente a prevalência e a severidade da maloclusão, devido ao menor acesso ao tratamento odontológico (predispõe a cárie e conseqüente perda precoce de dentes) e ao tratamento ortodôntico pelos indivíduos de baixa renda (dificultam o acesso a informação para prevenção de maloclusões, como por exemplo, os hábitos deletérios) (TOMITA et al., 2000). No entanto, as divergências encontradas nos estudos de prevalência estão mais relacionadas a raças e miscigenação (RIBAS et al., 2004; SULIANO et al., 2007) e, principalmente, a fatores como hábitos deletérios, desenvolvimento sócio demográfico, urbanização

\section{DISCUSSÃO}


(acesso a água fluoretada), nutrição e tamanho da família (FRAZÃO et al., 2002).

Outros fatores importantes a serem considerados são o delineamento dos estudos bem como a amostragem e o método de análise dos resultados que contribuem para as discrepâncias dos dados disponíveis sobre diferentes populações para além das diferenças de caráter étnico e sociodemográfico (RIBAS et al., 2004; THOMAZ \& VALENÇA, 2005).

Neste trabalho verificou-se que a mordida aberta foi muito frequente entre os escolares (35,01\%), concordando com os estudos como o de Sadakyo et al. (2004) que também constataram altos índices desta maloclusão (40,08\%), contrastando com outros estudos que encontraram 15\% (KABUE et al., 1995), 27,6\% (OLIVEIRA et al., 1998) e 15,06\% (THOMAZ \& VALENÇA, 2005). Os altos índices de mordida aberta nesta faixa etária estão relacionados principalmente a associação de hábitos bucais deletérios como a onicofagia, sucção não-nutritiva e o hábito de morder objetos (DE VIS et al., 1984; THOMAZ \& VALENÇA, 2005). Além disso, a mordida aberta apresentou uma correlação positiva e significante com a Classe II, ou seja, a presença de uma está associada à presença da outra.

A análise de uma amostra em diferentes faixas etárias demonstra que a mordida aberta anterior decresce de $66,7 \%$ aos três anos para $20 \%$ aos seis anos (DE VIS et al., 1984; OLIVEIRA et al., 1998; THOMAZ \& VALENÇA, 2005). No entanto, há relatos (SADAKYO et al., 2004) de prevalência desta maloclusão de $41,03 \%$ aos 4 anos e $45,83 \%$ aos seis anos, sendo que essas divergências podem ser justificadas devido ao fato de um possível prolongamento dos hábitos de sucção não nutritiva e nutritiva variáveis para cada amostra. Segundo Thomaz e Valença (2005), existe uma associação estatisticamente significante entre a mordida aberta e os hábitos parafuncionais.

Já em relação às mordidas profundas verificou-se que $19,84 \%$ das crianças examinadas a possuíam, sendo coerente com os valores encontrados por outros autores como Silva Filho et al. (1990) de 19,86\%, porém mais baixos do que encontrados por outros autores como Almeida et al. (2008) de 27\%.

Dentre os problemas transversais a atresia foi observado na maioria das crianças $(49,80 \%)$, ocorrendo com menos prevalência em outros estudos, com o de Sadakyo et al. (2004), cujo resultado foi de $12,55 \%$. Neste trabalho verificou-se uma importante correlação positiva e estatisticamente significante entre atresia e mordida cruzada, ou seja, a observação da mordida cruzada implica na presença de atresia. Além desta, a correlação entre atresia, mordida cruzada e mordida aberta foi estatisticamente significante $(p=0,0001)$, e também a correlação entre Classe II, mordida aberta, atresia e mordida cruzada ( $p=0,0001)$.

Com relação aos problemas de espaço, $63,42 \%$ não possuíam este tipo de maloclusão, $3,89 \%$ apresentavam perda severa de espaço, $12,06 \%$ tinham diastemas entre os dentes e $20,62 \%$ apresentavam cáries extensas o suficiente a ponto de haver perda mésio-distal do dente, com conseqüente perda de espaço. Os valores de cárie foram altos considerando que a cidade possui água fluoretada, inclusive na zona rural, enfatizando a necessidade de orientação de higienização e hábitos alimentares.

As correlações negativas e significantes entre cáries e diastemas encontrada neste trabalho demonstram que os escolares que possuíam diastemas não possuíam cáries que pudessem causar perda de espaço. Este fato pode ser explicado pela facilidade de higienização dos dentes na presença de diastemas. Lesões de cáries extensas não tratadas são fatores 
agravantes na determinação de maloclusões figurando a terceira posição na escala de prioridades e de problemas de saúde pública no Brasil (TOMITA et al., 2000).

A presença de cáries extensas pode levar a perda precoce do elemento dentário. Esta perda sem a imediata reposição ou manutenção do espaço é considerada um dos freqüentes fatores etiológicos de maloclusões (SEWARD, 1965), podendo acarretar mau posicionamento dos dentes adjacentes, extrusão do antagonista e desvios de linha mediana. As principais causas de perda precoce estão associadas à cárie dentária e trauma (ROSE, 1996). Segundo Ribas et al. (2004) este problema pode estar associado ao desenvolvimento de maloclusões, devendo ser levado em consideração no planejamento de medidas preventivas. No entanto, alguns autores consideram que a perda prematura de dentes decíduos não influencia no desenvolvimento da dentição e oclusão (KRONFELD, 1964; SEWARD, 1965).

Ao verificarmos as maloclusões sagitais, observou-se que a proporção de indivíduos portadores de Classe I $(56,80 \%)$ foi maior do que os portadores de Classe II (36,96\%) e de Classe III (6,22\%), concordando com os resultados de diversos outros estudos epidemiológicos (SILVA FILHO et al., 1990; TIPTON \& RINCHUSE, 1990; ROSE, 1996; ARASHIRO et al., 2009). Alguns autores (NORMANDO et al., 1999) verificaram uma maioria de escolares com maloclusão de Classe II, sendo que Ribas et al. (2004) verificou maior porcentagem de Classe II em grupos com pouca mestiçagem. Estas divergências também podem ser explicadas pelo fato de que alguns autores, como no caso deste trabalho, incluem a maloclusão de Classe I e a oclusão normal no mesmo grupo (ARASHIRO et al., 2009).

Diante da atual miscigenação da população brasileira faz-se necessário uma avaliação de cada região localizando as principais maloclusões encontradas para que um plano de tratamento e/ou orientação possam ser estudados juntamente com os órgãos públicos competentes para a realização de medidas que possam intervir e/ou interceptar as maloclusões. Assim, com este estudo pôde-se traçar um perfil das crianças residentes no município de Lavrinhas-SP com idade entre 4 e 7 anos para direcionamento das medidas necessárias a atender esta população, sugerindo um programa de prevenção incluindo orientação de higienização, de hábitos nutricionais e da remoção de hábitos parafuncionais em idade adequada.

\section{CONCLUSÃO}

Este levantamento ampliou 0 conhecimento sobre os problemas oclusais que acometem as crianças do município de Lavrinhas/SP, enfatizando a necessidade de tratamento ortodôntico, permitindo que orientações adequadas e direcionadas de higienização, nutrição e da remoção de hábitos parafuncionais sejam instituídos, já que um alto índice de maloclusão (91,05\%) pôde ser observado. Assim, a integralização da ortodontia nos serviços públicos de saúde é importante e, quando iniciada em fase precoce (preventiva/interceptadora), possui custos muito mais baixos viabilizando um possível tratamento.

\section{REFERÊNCIAS}

Almeida-Pedrin RR, Silva EE, Ferreira FPC, Almeida MR. Prevalência das más-oclusões em jovens de seis a doze anos de idade na cidade de Miranda/MS. Ortodontia SPO. 2008;41(4):384-92.

Angle EH. Classification Malocclusion. Dental Cosmos. 1989;41:248-64, 350-7.

Arashiro C, Ventura MLS, Mada EY, Uenshi PT, Barbosa JA, Bonecker MJS. Prevalência de maloclusão em escolares do município de Campinas. RGO. 2009;57(4):407-11.

Bresolin D. Controle e prevenção da má oclusão. In: Saúde bucal coletiva. São Paulo: São Paulo, 2000. Cap. 8, p.473-9. 
De Vis $\mathrm{H}$, De Boever JA, Van Cauwenbergue $\mathrm{P}$. Epidemiologic survey of funtional conditions of the masticatory system in Belgian children aged 3-6 years. Community Dent Oral Epidemiol. 1984;12(3):203-7.

http://dx.doi.org/10.1111/i.1600-

0528.1984.tb01440.x

Foster TD, Menezes DM. The assessment of occlusal features for public health plannig purposes. Am J Orthod. 1976;69(1):83-90. http://dx.doi.org/10.1016/0002-9416(76)90100-7

Frazão P, Navai PC, Latorre MRDO, Castellanos RA. Prevalência de oclusopatia na dentição decídua e permanente de crianças na cidade de São Paulo, Brasil, 1996. Cad Saúde Pública. 2002;18(5):1197-205.

IBGE. IBGE Cidades - Lavrinhas, 2007. Disponível em <http://www.ibge.gov.br/cidadesat/painel/painel.ph p?codmun=352660\#>. Acesso em: 05 nov. 2010.

Kabue MM, Moracha JK, Ng'ang'a PM. Malocclusion in children aged 3-6 years in Nairobi, Kenya. East Afr Med J. 1995;72(4):210-2.

Kronfeld S. Factors of occlusion as they affect space maintenance. J Dent Child. 1964;31(4):302-13.

Marques LS, Barbosa CC, Ramos-Jorge ML, Pordeus IA, Paiva SM. Prevalência da maloclusão e necessidade de tratamento ortodôntico em escolares de 10 a 14 anos de idade em Belo Horizonte, Minas Gerais, Brasil: enfoque psicossocial. Cad Saúde Pública. 2005;21(4):1099-106.

http://dx.doi.org/10.1590/S0102311X2005000400012

Normando ADC, Brandão AM, Matos JN, Cunha AV, Mohry O, Jorge ST. Má oclusão e oclusão normal na dentição permanente - um estudo epidemiológico em escolares do município de Belém, PA. Rev Paraense Odontol. 1999;4(1):216.

Oliveira AGRC, Unfer B, Costa ICC, Arcieri RM, Guimarães LOC, Saliba NA. Levantamentos epidemiológicos em saúde bucal: análise da metodologia proposta pela Organização Mundial da Saúde. Rev Bras Epidemiol. 1998;1(2):177-89.

Projeto SB Brasil 2003: Condições de saúde bucal da população brasileira 2002-2003: resultados principais. Ministério da saúde, Secretaria de Atenção à Saúde, Departamento de Atenção Básica, 2004. 68p.
Ribas MO, Orellana B, Fronza F, Gasparim GR, Mello GS, Simas Neta MLS, Kowalski RV, Araújo RC. Estudo epidemiológico das maloclusões em escolares de 06 a 08 anos na cidade de Curitiba, Paraná. RSBO. 2004;1(1):22-9.

Rose JS. Early loss of teeth in children. Brit Dent J. 1996;120:275-80.

Sadakyo CA, Degan VV, Pignataro Neto G, Puppin Rontani RM. Prevalência de má oclusão em pré-escolares de Piracicaba-SP. Ciênc Odontol Bras. 2004;7(2):92-9.

Seward SF. Natural closure of deciduous molar extraction spaces. Angle Orthod. 1965;35(1):2932.

Silva Filho OG, Freitas SF, Cavassan AO. Prevalência de oclusão normal e má oclusão em escolares da cidade de Bauru (São Paulo). Parte I: relação sagital. Rev Odontol Univ São Paulo. 1990;4(2):130-7.

Suliano AA, Rodrigues MJ, Júnior AFC, Fonte PP, Porto-Carreiro CF. Prevalência de maloclusão e sua associação com alterações funcionais do sistema estomatognático entre escolares. Cad Saúde Pública. 2007;23(8):1913-23. http://dx.doi.org/10.1590/S0102$\underline{311 \times 2007000800018}$

Thomaz EBAF, Valença AMG. Prevalência de má-oclusão e fatores relacionados à sua ocorrência em pré-escolares da cidade de São Luís, MA, Brasil. 2005;12(2):212-21.

Tipton TR, Rinchuse DJ. The relationship between static occlusion and functional occlusion in a dental school population. Angle Orthod. 1990;61(1):57-63.

Tomita NE, Bijella VT, Franco LJ. Relação entre hábitos bucais e má oclusão em pré-escolares. Rev Saúde Pública 2000;34:299-303. 